\title{
Specialised sensorial block
}

\author{
Mihai Avram ${ }^{1, *}$, Constantin Bucsan ${ }^{1}$, and Tudor Cătălin Apostolescu ${ }^{2}$ \\ ${ }^{1}$ Politehnica University of Bucharest, Department of Mechatronics and Precision Mechanics, \\ SplaiulIndependentei nr. 313, Bucharest, Romania \\ ${ }^{2}$ Titu Maiorescu University of Bucharest, Faculty of Informatics, Department of Informatics, \\ CaleaVacaresti nr. 187, Bucharest, Romania
}

\begin{abstract}
In driving systems using working fluids, for monitoring the fluid's parameters such as pressure, flow and temperature appropriate transducers are present in different areas of the system. Such an approach has certain disadvantages with regard to the installation and powering of the transducers as well as the transmission of the signals supplied by the transducers to the system's electronics command block. Both hydraulic and pneumatic driving systems are considered. The paper proposes a sensor block which integrates the above mentioned sensors and eliminates the previously outlined disadvantages. The proposed block can be designed for an easy installation in a driving system containing a pneumatic linear motor; basically for a standardised motor one of the caps is replaced with the proposed block. An example is also shown for the integration of the proposed block in the structure of a pneutronic system.
\end{abstract}

\section{Introduction}

Production systems in the future will be based on mechatronic assemblies with autonomous functioning; these are also known as intelligent components. Data processing is increasingly handled at a local level with a higher number of functions being directly integrated into modules. These communicate in a network and can organise and selfconfigure for receiving commands from the superior control level. These aspects outline a new stage of industrial development, called Industry 4.0, with components defined by 4 ,i” properties:

- intuitive operation;

- intelligent: due to functional integration, such as a programmable logical controller with communication abilities;

- internet: local network communication and Internet connection abilities, by using WLAN or industrial Ethernet;

- integrators: modules that $\log$ on to the main computer, communicate available capabilities and so their actions can be planned in the production process.

Preconfigured controllers and adaptable interfaces minimise installation times for the system's installation, configuration and integration. Intelligent components have a "plug and produce" capability. This principle is similar to a USB computer interface where

\footnotetext{
*Corresponding author: mavram02@yahoo.com
} 
connected devices can $\log$ on and communicate directly with a computer. In future enterprises, individual components, production subsystems and modules will be able to similarly log in to the production system [1].

In Industry 4.0, smart factories represent a combination of the following components [2, 9]:

- Internet of Services, or Cloud Computing, that allows for the usage of applications, data bases, records, storage and computing power for all those connected to a network;

- Cyber-physical systems, for digital and physical connectivity;

- Internet of Things (IoT) is the most important and active of these components, where connected devices use the Internet without any human interference.

As with all aspects concerning production, hydraulic and pneumatic technologies will also benefit from the Industry 4.0 standards. A study of the Rexroth Bosch Group shows that most controllers for hydraulic equipment are pre-programmed with the necessary software for passing from "path control" to "force control", in order to achieve synchronization on the production line. This gives hydraulic equipment a decentralised intelligence which allows for autonomous operation and adjustment to the changing process parameters [3].

As for pneumatic equipment, FESTO has been simulating process and factory automation for some years now and offers a wide variety of products for control technologies and software by using smart components to simplify and shorten the configuration and operation of engineering processes [1].

As for the development of equipment and hydraulic and pneumatic driving systems a strong trend toward computerisation can be noticed in order to achieve real time system and equipment control using programmable controllers. This obviously involves that the system contain sensor elements for the physical parameters needed in the application. Also, a low energy consumption is required and the possibility to intervene on the functioning system in order to reduce idle times caused by stops.

\section{Analysis of the options for integration of sensors in the structure of pneumatic and hydraulic equipment}

Below we have 3 scenarios which led the authors to the idea of developing intelligent sensor modules that integrate more transducers.

Scenario 1 refers to pneumatic/hydraulic systems where different measurements need to be made, such as: pressure, pressure difference, flow and temperature. Transducers need to be placed at several points in the system. A more efficient option would be grouping these transducers into a single module, universally and efficiently developed so that it involves a single power source and a single communication protocol with or without wire connection.

Figure 1 shows a hydraulic positioning system from the Automation and Robotics Lab in the Department of Mechatronics and Precision Mechanics at the Politehnica University of Bucharest [4]. The load positioning is done with micrometric accuracy with a specifically designed software. Real time information is processed from a series of pressure, flow and position sensors which allow for interference compensation. The system is driven by a PC and interfaced with Field Point input/output modules (figure 2). The command program is developed using the graphic programming language Lab VIEW.

This system uses different transducers located along the circuit that are produced by different manufacturers pressure transducer $\mathrm{T}_{P}$, flow transducer $\mathrm{T}_{q}$, position transducer $\mathrm{T}_{p o z, l}$.

The main challenge consists in coupling these transducers to the hydraulic system and the driving system. The existence of a block module that includes all these sensors with a 
single power supply and communication interface could result in a simpler system design process and an optimal control for the transducers block.

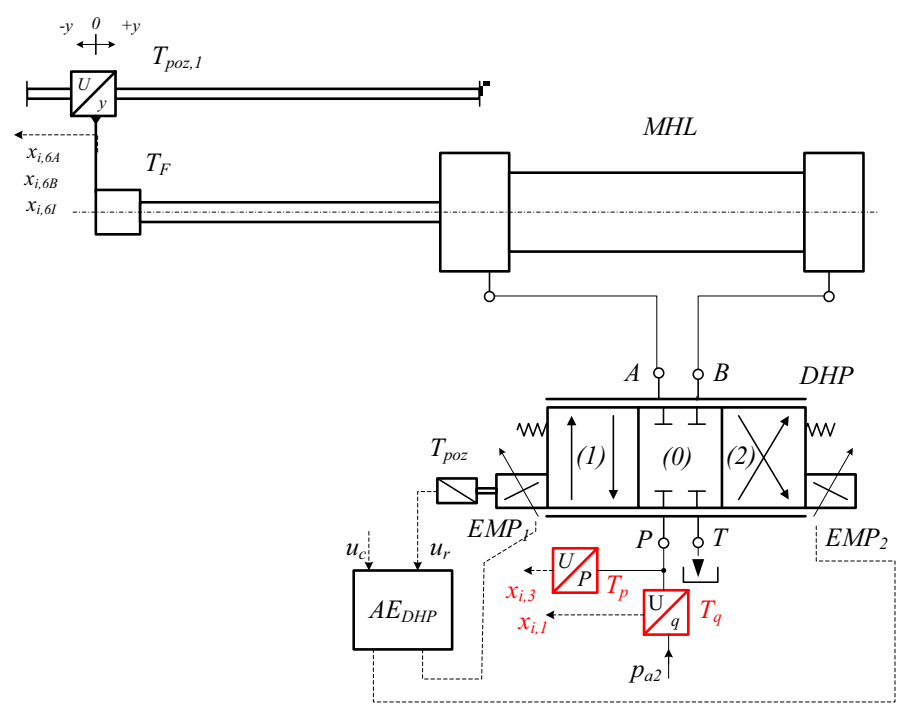

Fig. 1. Hydraulic positioning system.

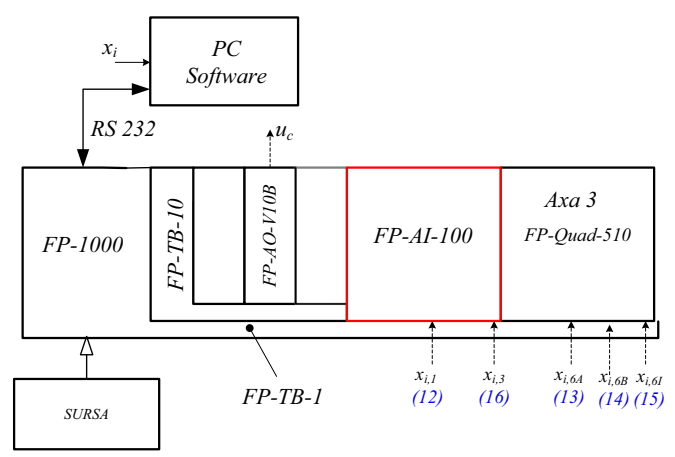

Fig. 2. PC Interfaced with Field Point input/output modules.

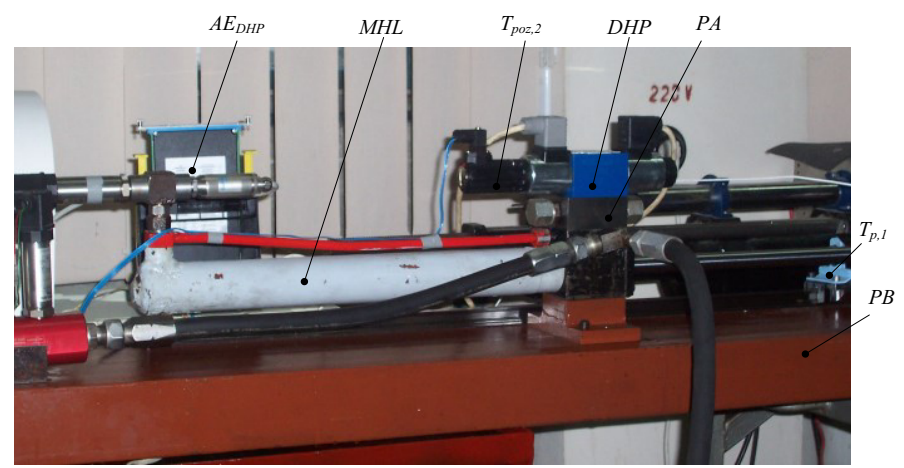

Fig. 3. Experimental model developed by the authors.

Scenario 2 involves proportional equipment. A reoccurring problem for this equipment is represented by hysteresis. The global hysteresis can be significantly reduced by automatic adjustment for the position of the mobile fixture [5]. An inductive position 
reaction is generally used. A practical example for a proportional distributor could be the one produced by Rexroth (figure 4), this being equipment4 WREE 6 EA08 [3].

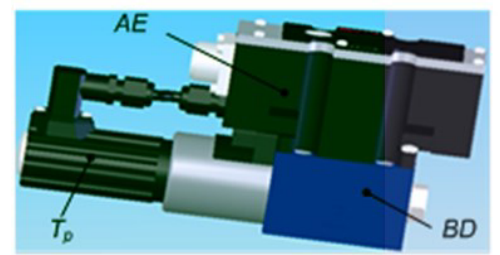

Fig. 4. Rexroth proportional distributor

Similar situations can be encountered for other manufacturers in the area of pneumatic systems such as FESTO and SMC.

Scenario 3 follows the construction of hydraulic motors used in positioning systems. These have position transducers placed on the outside of the motor (resistive, temposonic), or integrated (magneto-strictive). These solutions are available from several manufacturers but the costs are significant and also, they require specialised command modules. Using a different command system than the one imposed by the manufacturers can prove difficult and costly. An example for a hydraulic motor produced by Rexroth [3] is shown in figure 5 , with the magneto-strictive transducer 8integrated in the motor's construction and the distribution equipment 7 placed on the motor's stator.

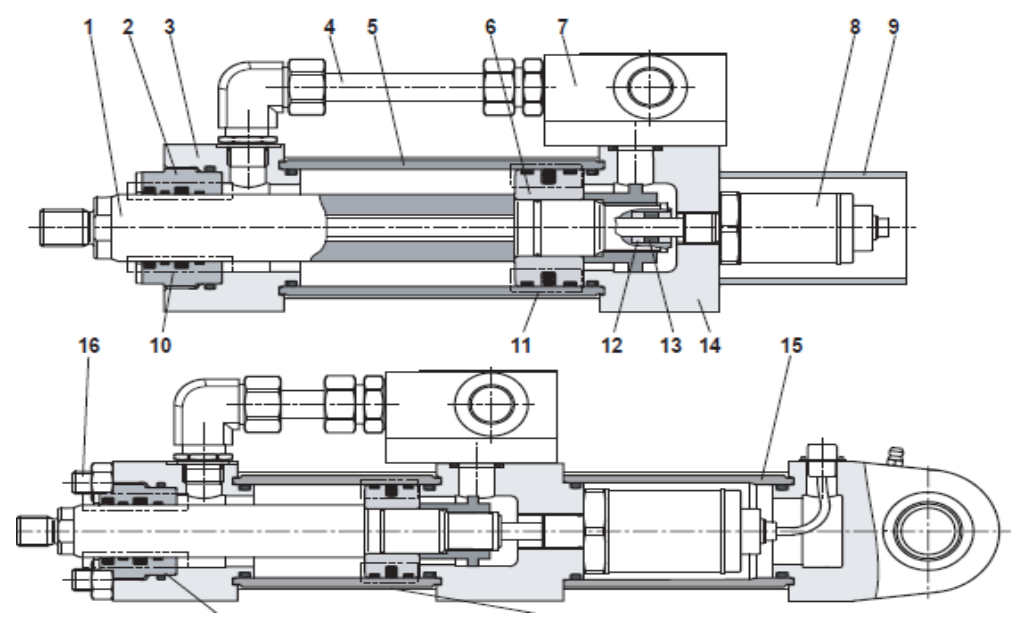

Fig. 5. Rexroth hydraulic motor.

If one wishes to use the motor with a different controller than the one indicated by the manufacturer problems arise regarding the interface and communication protocol. This is another reason for which an intelligent module that can adapt to any motor with a simple, clear and universal interface could prove to be a very efficient solution.

\section{Theproposed solution}

The authors have developed the structure for an intelligent sensor module, with integration capabilities for most pneumatic equipment and systems with the imposed performance criteria.

Figure 6 shows the basic schematics for the proposed sensor module. 


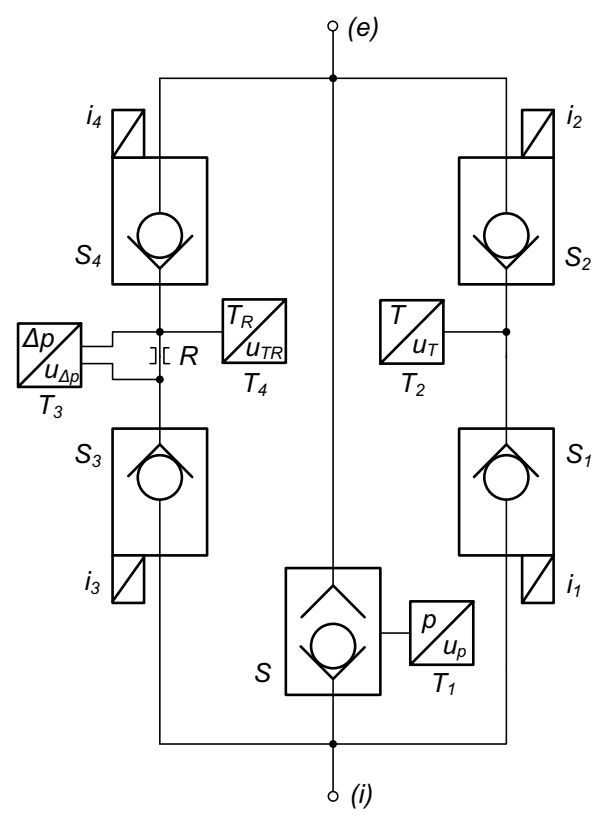

Fig. 6. Schematics for the proposed sensor module.

The sensor block consists of the controlled valves $S_{1}, S_{2}, S_{3}$ and $S_{4}$, that according to the command currents $i_{1}, i_{2}, i_{3}$ and $i_{4}$, select the work fluid's direction of flow so that its main parameters can be measured: pressure $p$, temperature $T$ and mass flow $\dot{m}$.

Flowmis calculated based on values obtained by direct measurement of the pressure difference $\Delta p$ on the calibrated sectionRand of the fluid's temperature $T$.

The formula based on which these calculations are done is [6]:

$$
\dot{m}=\frac{K \cdot P_{a} \cdot S_{n}}{\sqrt{T_{a}}} \cdot N\left(\frac{P}{P_{a}}\right)
$$

where:

$$
\begin{aligned}
& P=\left\{\begin{array}{l}
P_{i} \text { for the flow direction }(i) \rightarrow(e) \\
P_{e} \text { for the flow direction }(e) \rightarrow(i)
\end{array}\right. \\
& N\left(\frac{P-\Delta p}{P}\right)=\left\{\begin{array}{lr}
1 & \text { if } 0 \leq \frac{P-\Delta p}{P} \leq 0,528 \\
a \cdot\left[\left(\frac{P}{P_{a}}\right)^{\frac{2}{\chi}}-\left(\frac{P}{P_{a}}\right)^{\frac{(\chi+1)}{\chi}}\right]^{\frac{1}{2}} & \text { if } \frac{P-\Delta p}{P}>0,528
\end{array}\right.
\end{aligned}
$$

The following relevant coefficients are considered:

$$
\begin{gathered}
\chi=1,4[-] \\
R=287,04\left[\frac{m^{2}}{s^{2} \cdot K}\right] \\
K=0,04042\left[\frac{\sqrt{K} \cdot s}{m}\right] \\
a=2,6143[-]
\end{gathered}
$$


So for the flow direction from (i) to (e), transducer $T_{l}$ measures the pressure $p_{i}$. If valve $S_{1}$ is commanded, the work fluid flows through and transducer $T_{2}$ measures the temperature $T_{i}$. If valve $S_{3}$ is commanded, the work fluid flows through the resistance $R$ and transducer $T_{3}$ measures the pressure drop $\Delta p$ on this resistance, and transducer $T_{4}$ measures the fluid's temperature in the resistance's area $T_{R}$. Considering these values the flow $q$ for the work fluid can be calculated.

Table 1 shows a synthesis of the system's functioning.

Table 1. Synthesis of system's functioning.

\begin{tabular}{|c|c|c|c|c|c|c|c|}
\hline $\begin{array}{c}\text { Flow } \\
\text { direction }\end{array}$ & $\boldsymbol{i}_{\boldsymbol{1}}$ & $\boldsymbol{i}_{\boldsymbol{2}}$ & $\boldsymbol{i}_{3}$ & $\boldsymbol{i}_{\boldsymbol{4}}$ & $\boldsymbol{p}$ & $\boldsymbol{T}$ & $\boldsymbol{q}=\boldsymbol{f}\left(\boldsymbol{\Delta p}, \boldsymbol{T}_{\boldsymbol{R}}\right)$ \\
\hline \multirow{2}{*}{$(i) \rightarrow(e)$} & 1 & 0 & 0 & 0 & $p_{i}$ & $T_{i}$ & - \\
\cline { 2 - 8 } & 0 & 0 & 1 & 0 & $p_{i}$ & - & $q_{i}$ \\
\hline \multirow{2}{*}{$(e) \rightarrow(i)$} & 0 & 1 & 0 & 0 & $p_{e}$ & $T_{e}$ & - \\
\cline { 2 - 8 } & 0 & 0 & 0 & 1 & $p_{e}$ & - & $q_{e}$ \\
\hline
\end{tabular}

The construction for the controlled valve is shown in figure $7 \mathrm{a}$.

The valve is composed of the body 1 , the cover 2 , where the valve's seat is machined, the valve 3 , solidary with the rigid centre 4 of the cut-out metallic membrane 5 , the cylindrical helical spring 6 , that presses the valve on the seat, and the electromagnet 7 .

The cut-out metallic membrane is shown in figure $7 \mathrm{~b}$.

(2)
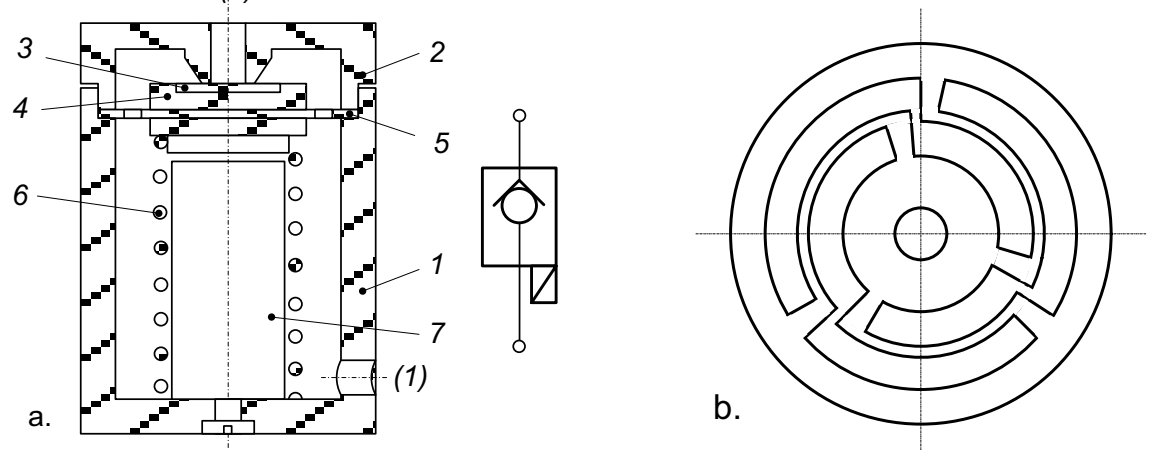

Fig. 7. a. Construction of the controlled valve; b. Cut-out metallic membrane.

If the work fluid enters through hole (1), its pressure acts as the spring in the direction in which the valve presses on the seat, so the controlled valve is closed.

If a command current is applied on the electromagnet this pulls the rigid centre of the membrane overcoming the force in the spring and so the controlled valve is opened allowing the fluid to exit through hole (2).

If the work fluid enters through hole (2), its pressure creates a force that overcomes the force in the spring, the rigid centre of the membrane with its valve will move away from the chair and the fluid will flow through the controlled valve and will exit through hole (1).

Based on the schematics in figure 6 the authors designed the experimental 3D model. The model should be easily integrated in the construction of a linear pneumatic motor where it would replace one of its covers. 


\section{An example of integrating the sensor block in a pneutronic system}

Figure 8 shows a pneutronic system containing the following equipment:

- MPL - Linear pneumatic motor with magnetic couplings and integrated position transducer with one of the lids replaced by the proposed sensor block;

- $\quad D P P$-proportional pneumatic distributor;

- $\quad B E C$ - electronic command block.

The system allows for multiple functions, such as:

- positioning a load with specific precision at any point of the work run [5];

- data acquisition for functional parameters (pressures, temperatures, mass flows) and their comparison with data obtained from numeric simulation; this ensures verification for the developed mathematical model;

- adjustment of load speed at the desired value and keeping that speed constant even with the resistant force varying over time.

The proposed system has a simple structure, is a multifunctional system and the command electronics is implemented in the system's construction.

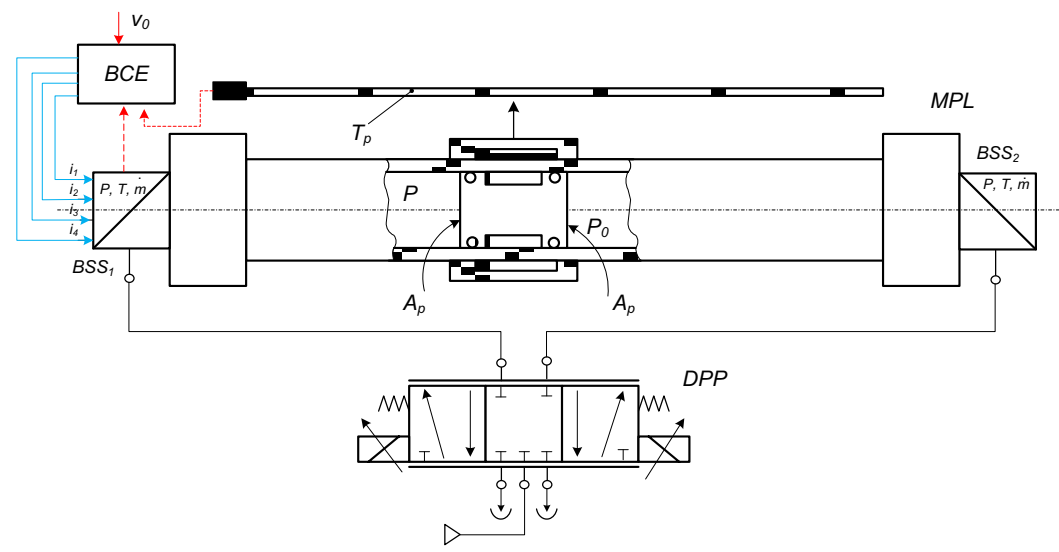

Fig. 8. Pneutronic system.

An example for an algorithm developed for the last function is shown next - load speed adjustment at the desired value and keeping constant that speed even if the resistant force varies over time.

This can be done by modifying in real time the control value $i$ for the proportional pneumatic distributor $D P P$ so that the desired speed can be maintained.

In order to determine the necessary correction $\Delta i$ the starting point is the mathematical model for the linear pneumatic motor $M P L$. This model consists of the following equations: - the motion equation of the mobile subassembly:

$$
M_{r} \cdot \frac{d v}{d t}=\left(P-P_{0}\right) \cdot A_{p}-F(t)
$$

where: $M_{r}$ - reduced mass of the mobile subassembly (piston, mass, load),

$F(t)$ - resisting force.

- the differential equation of the pressure in the motor's active chamber:

$$
\frac{d P}{d t}=\frac{\chi \cdot R \cdot T_{a}}{V_{0}+A_{p} \cdot x} \cdot \dot{m}-\frac{\chi \cdot P \cdot A_{p}}{V_{0}+A_{p} \cdot x} \cdot v
$$


Considering a constant speed movement: $v=v_{0}=c t$. și $\frac{d v}{d t}=0$ the motion equation becomes:

$$
P-P_{0}=\frac{F(t)}{A_{p}}
$$

By deriving:

$$
\frac{d P}{d t}=\frac{1}{A_{p}} \cdot \frac{d F(t)}{d t}
$$

By replacing relation (5) in relation (4):

$$
\dot{m}=\frac{V_{0}+A_{p} \cdot x}{\chi \cdot R \cdot T_{a} \cdot A_{p}} \cdot \frac{d F(t)}{d t}+\frac{P \cdot A_{p}}{R \cdot T_{a}} \cdot v
$$

Following measurements values are determined at any time $P=P_{i}, T_{a}=T_{i}$ and position $\mathrm{x}$. Using relation (6) the necessary flow for obtaining the desired speed can be determined $v=v_{0}$. At the same time by measuring the real mass flow $\dot{m}_{r}$ for powering the active chamber, corrections for the control values of the pneumatic proportional distributor can be established:

$$
\Delta i=\frac{\left|\dot{m}-\dot{m}_{r}\right|}{k_{D P P}}
$$

Where $k_{D P P}$ is the proportionality factor for the proportional pneumatic directional valve.

\section{Conclusions}

Placing the necessary transducers for monitoring the work fluid parameters (pressure, flow and temperature) for an integrated sensor block in the construction of the execution element eliminates the disadvantages associated with transducer installation, powering and signal transmission from the transducers to the electronic command block for the respective system. The authors have developed a multifunctional driving system containing the proposed sensor module. This system has a particularly simple structure with the command electronic implemented in its build and the command aspects ensured by a controller based electronic system.

\section{References}

1. $\quad * * *$ www.festo.com, Accesed 18.05.2017

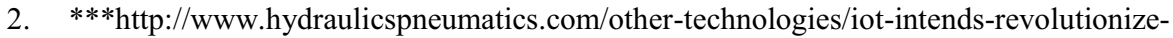
hydraulics-and-pneumatics, Accesed 8.02.2017

3. ***www.boshrexroth.com, Accesed 1.03.2017

4. M. Avram, C.Bucsan, Sisteme de actionare pneumatice inteligente, (EdituraPolitehnica PRESS, Bucuresti, 2014)

5. M. Avram, D. Duminica, C. Udrea, V. Gheorghe, Hidronică și pneutronică - Aplicatii, (EdituraUniversitară, Bucuresti, 2008)

6. M. Avram, Actionări hidraulice si pneumatice - Echipamente si sisteme clasice si mecatronice, (EdituraUniversitară, Bucuresti, 2005)

7. M. Avram, C. Bucsan, V. Constantin, Buletinul Institutului Politehnic din Iaşi, LVI(LX), 1 (2010)

8. S. Hesse, Blue Digest on Automation, Sensors in Production Engineering, Festo AG \& Co, (Available at: http://www.festo-didactic.com/int-en/seminarsearch/?fbid=aW50LmVuLjU1Ny4xNy4yOA\&search=download, 2001)

9. G. Belforte, G. Eula, Journal of Control Engineering and Applied Informatics, 14(4), 70 (2012) 